\title{
Parcellation of the Thalamus Using Diffusion Tensor Images and a Multi-object Geometric Deformable Model
}

\author{
Chuyang Ye ${ }^{a}$, John A. Bogovic ${ }^{a}$, Sarah H. Ying ${ }^{b}$, and Jerry L. Prince ${ }^{a}$ \\ ${ }^{a}$ Department of Electrical and Computer Engineering, Johns Hopkins University, Baltimore, \\ MD, USA 21218; \\ ${ }^{b}$ Departments of Radiology, Neurology, and Ophthalmology, Johns Hopkins University School \\ of Medicine, Baltimore, MD, USA 21287
}

\begin{abstract}
The thalamus is a sub-cortical gray matter structure that relays signals between the cerebral cortex and midbrain. It can be parcellated into the thalamic nuclei which project to different cortical regions. The ability to automatically parcellate the thalamic nuclei could lead to enhanced diagnosis or prognosis in patients with some brain disease. Previous works have used diffusion tensor images (DTI) to parcellate the thalamus, using either tensor similarity or cortical connectivity as information driving the parcellation. In this paper, we propose a method that uses the diffusion tensors in a different way than previous works to guide a multiple object geometric deformable model (MGDM) for parcellation. The primary eigenvector (PEV) is used to indicate the homogeneity of fiber orientations. To remove the ambiguity due to the fact that the PEV is an orientation, we map the PEV into a 5D space known as the Knutsson space. An edge map is then generated from the 5D vector to show divisions between regions of aligned PEV's. The generalized gradient vector flow (GGVF) calculated from the edge map drives the evolution of the boundary of each nucleus. Region based force, balloon force, and curvature force are also employed to refine the boundaries. Experiments have been carried out on five real subjects. Quantitative measures show that the automated parcellation agrees with the manual delineation of an expert under a published protocol.
\end{abstract}

Keywords: thalamic parcellation, DTI, 5D Knutsson space, multiple object geometric deformable model

\section{INTRODUCTION}

The thalamus is a symmetrical gray matter structure in the human brain that serves as a relay station for sensory information. It is divided into nuclei, many of which receive different sensory signals and, after some processing, send the signals on to particular parts of the cerebral cortex. Other nuclei receive input signals from the cortex and relay them to different cortical regions. The thalamus consists of about 50 nuclei, ${ }^{1}$ a coarse grouping of which is illustrated in Figure 1. Since nuclei-specific neurodegeneration is observed in some diseases, such as multiple sclerosis (MS), ${ }^{2}$ the ability to automatically parcellate the thalamic nuclei could lead to enhanced diagnosis or prognosis in patients with brain disease.

Because conventional anatomic MR images do not provide sufficient contrast between the thalamic nuclei, previous published works on the thalamic parcellation have used diffusion tensor images (DTI). Apart from connectivity information, ${ }^{3}$ most works have utilized tensor or fiber orientation homogeneity to parcellate the thalamus. ${ }^{4-9}$ Wiegell et al. ${ }^{4}$ used the combined spatial location and raw tensor as the features in $k$-means clustering, requiring a manual identification of cluster-to-nucleus correspondence as the final step; Duan et al. ${ }^{5}$ used a mean shift clustering instead of the $k$-means algorithm to improve the segmentation; Jonasson et al. ${ }^{6}$ adopted a level set method to segment the nuclei using a region force defined by the similarity of the tensors on each nucleus; Rittner et al. ${ }^{7}$ exploited the concept of tensorial morphological gradient and labeled the nuclei with a watershed method. In the work of Ziyan et al., ${ }^{8}$ several similarity measures were compared and it is concluded that using the primary eigenvector (PEV) which represents the fiber orientation is sufficient to indicate the homogeneity and more accurate than the other choices for the task of the thalamic parcellation. Fan et al. ${ }^{9}$ gave a first attempt to segment the thalamic nuclei with a multiple object geometric deformable model (MGDM) framework using the PEV on one subject without a quantitative validation. That work showed the potential for combining the MGDM framework and the PEV to accomplish thalamic parcellation.

Medical Imaging 2013: Image Processing, edited by Sebastien Ourselin, David R. Haynor,

Proc. of SPIE Vol. 8669, 866909 - @ 2013 SPIE - CCC code: 1605-7422/13/\$18

doi: $10.1117 / 12.2006119$

Proc. of SPIE Vol. 8669 866909-1 


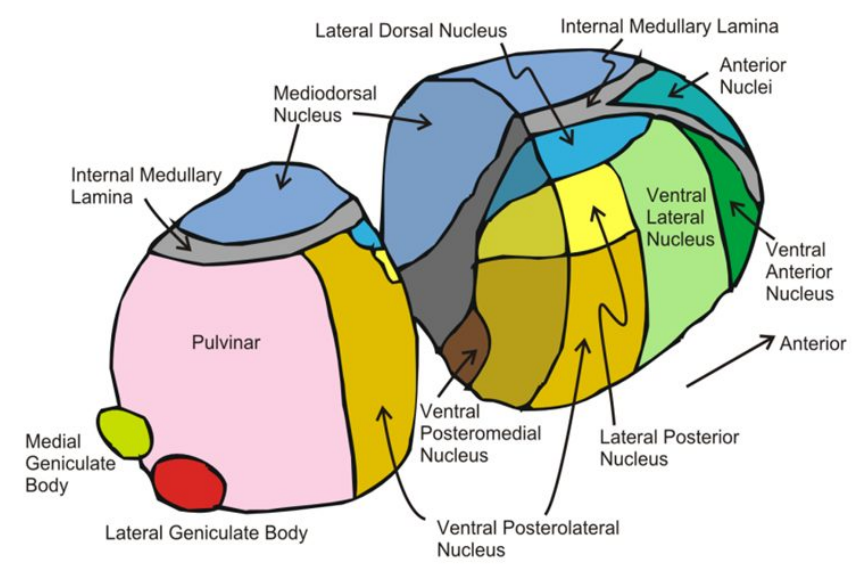

Figure 1. A schematic of the thalamic nuclei.

In this work, we propose a method for the thalamic parcellation within the MGDM framework ${ }^{9}$ that utilizes the PEV in a way that is different than previous works. MGDM segments multiple objects simultaneously and efficiently and can keep object relationships fixed. It is also a flexible framework which allows the incorporation of various forces designed for different purposes. In our method, unlike the work of Ziyan et al. ${ }^{8}$ and Fan et al., ${ }^{9}$ the PEV is not used directly to represent the homogeneity and develop the forces. Firstly, we map it into a $5 \mathrm{D}$ space known as the Knutsson space ${ }^{10}$ to eliminate the ambiguity brought about by the fact that the PEV is an orientation, not a direction. Then an edge map can be obtained from the 5D vector to show the divisions of the nuclei. Instead of seeking to segment regions that have similar fiber orientations, as in the work of Ziyan et al. ${ }^{8}$ and Fan et al., ${ }^{9}$ we make attempts to locate the boundaries of each nucleus on the computed edges. This is because some large nucleus can be composed of smaller nuclei which have different fiber orientations. If the PEV homogeneity is used directly, it is possible that the nucleus cannot be segmented properly; however, if we use the edge to locate the boundaries, with an appropriate initialization, the inhomogeneity within a large nucleus or nuclear group will not affect the parcellation. The rest of the paper is organized as follows: Section 2 introduces our method in detail; Section 3 shows the experiment on real subjects using our method; and finally Section 4 concludes the paper and discusses future work.

\section{METHOD}

In this work, we segment four nuclei or nuclear groups on each side of the thalamus: the anterior nuclear group (AN), the medial dorsal nucleus (MD), the ventral nuclear group (VN), and the pulvinar (PUL). A background (BG) label is also used. These four nuclei are selected because they have been reported to be visible in DTI, and have been manually segmented using a protocol that we previously developed. ${ }^{11}$ Their relative relationship is shown in Figure 2(a). In this section, we first introduce the mapping of the PEV into the 5D Knutsson space ${ }^{10}$ and the generation of the edge map. Then we describe the MGDM framework, the initialization, and the design of the forces that drives the evolution of the nuclei. Forces such as advection force, region force, balloon force, and curvature forces are used, and the role of these forces will be made explicit.

\subsection{Mapping PEV into Knutsson Space}

The PEV indicates the primary diffusion direction. It has been assumed that the PEV's exhibit orientation differences in different nuclei. ${ }^{8,9}$ However, since the PEV is an orientation rather than a direction, two opposite PEV's, for instance $\frac{1}{\sqrt{3}}(1,1,1)$ and $\frac{1}{\sqrt{3}}(-1,-1,-1)$, are equivalent. To address this ambiguity, for a PEV, $u=\left(u_{1}, u_{2}, u_{3}\right)$, we map it into the $5 \mathrm{D}$ Knutsson space using the following formula. ${ }^{10}$

$$
v=\left(v_{1}, v_{2}, v_{3}, v_{4}, v_{5}\right)=M(u)=\frac{1}{\|u\|}\left(u_{1}^{2}-u_{2}^{2}, 2 u_{1} u_{2}, 2 u_{1} u_{3}, 2 u_{2} u_{3}, \frac{1}{\sqrt{3}}\left(2 u_{3}^{2}-u_{1}^{2}-u_{2}^{2}\right)\right)
$$


This transformation maps the two 3D vectors with identical orientations to the same $5 \mathrm{D}$ vector and eliminates the ambiguity. With the $5 \mathrm{D}$ vector, we are able to compute an edge map of the orientation. First we estimate the gradient matrix $G$ by applying a finite difference operator, and then the Frobenius norm of $G$, i.e. $\|G\|_{F}$, are taken to get the edge map. ${ }^{11}$ An example of the edge map in the thalamus is shown in Figure 3(a).

\subsection{MGDM}

MGDM is a multiple object segmentation framework that guarantees no object overlap or gaps between objects. It can apply the forces that are conventionally used in geometric deformable models. ${ }^{9}$ Moreover, MGDM can apply these forces on the specific boundaries between designated objects rather than a uniform force on the whole boundary of each object, as shown in Equation 2.

$$
\frac{\partial \phi_{i, j}}{\partial t}+\left(f_{\text {reg: } i, j}+f_{\text {bal: } i, j}\right)\left|\nabla \phi_{i, j}\right|+f_{\text {adv }: i, j} \cdot \nabla \phi_{i, j}=\epsilon \kappa\left|\nabla \phi_{i, j}\right|
$$

Here $\phi_{i, j}$ is the level set function for the boundary between a certain object pair of interest, $i$ and $j . \kappa$ is the curvature, $f_{\text {reg:i,j }}$ and $f_{\text {bal:i, } j}$ stands for the region force and the balloon force respectively, and $f_{\text {adv: } i, j}$ is the advection force. We will take advantage of this capability by designing the forces for these specific boundaries. Besides, it is computationally efficient since it evolves only three distance functions and three label functions in 3D. ${ }^{9}$ As shown in Figure 1 and 2(a), the thalamic nuclei are disjoint objects that comprise the thalamus, thus MGDM is ideally suited for the thalamic parcellation task.

\subsection{Initialization}

To initialize MGDM, we use a single-atlas registration strategy. A manual delineation of the four nuclei was performed on one subject. Then for each subject to process, a manual mask of the thalamus is generated with the FA map according to a published protocol, ${ }^{11}$ and the manual delineation of the nuclei is affinely registered to the target with respect to the thalamus using the corresponding MP-RAGE images. An example of the initialization is shown in Figure 3(b) and it is overlaid on the edge map in Figure 3(c). We can see that the initialization is located in a reasonable place yet requires further refinement to achieve a good segmentation.

\subsection{Force Design}

In order to refine this initial alignment of thalamic nuclei, forces that will position the objects and their edges according to the underlying data must be designed. The generalized gradient vector flow (GGVF) $f_{\text {GGVF }}$ is calculated from the edge map by solving Equation 3.

$$
f_{\mathrm{GGVF}}=\underset{f}{\arg \min } \int_{\Omega} g(|\nabla f|)|\nabla \vec{v}|^{2}+h(|\nabla f|)|\vec{v}-\nabla f|^{2} \mathrm{~d} \omega
$$

where $g(|\nabla f|)=e^{-|\nabla f| / K}, h(|\nabla f|)=1-g(|\nabla f|)$, and $\vec{v}$ is the gradient field of the edge map. ${ }^{12}$ The GGVF plays a major role in our method, because it is the force that utilizes the underlying DTI data to drive the boundaries of the nuclei as the advection force. Besides the GGVF force, a region force $f_{\text {reg }}$ is used to ensure the collection of nuclei to fill the thalamus; a balloon force $f_{\text {bal }}$ on the MD enables the nucleus to grow in order to be influenced by the GGVF force; and finally a curvature force $\kappa$ is applied to preserve smoothness. A diagram of the forces on each nuclear boundary is shown in Figure 2(b) for overview. The application of these forces in the MGDM framework are to be introduced in detail in the following paragraphs.

As mentioned above, MGDM can apply different forces on the boundaries between different object pairs. Here we denote the force on the boundary between object $i$ and $j$ as $f_{i, j}$, where $i, j \in\{\mathrm{BG}, \mathrm{AN}, \mathrm{MD}, \mathrm{VN}, \mathrm{PUL}\}$. The GGVF force is applied on each boundary between the nuclei as the advection force $f_{\text {adv:i,j }}$, i.e.,

$$
f_{\mathrm{adv}: i, j}=\left\{\begin{array}{l}
0, \quad \text { if } \quad i \text { or } j=\mathrm{BG} \\
f_{\mathrm{GGVF}}, \quad \text { otherwise }
\end{array} .\right.
$$

An example of the GGVF is overlaid on the edge map axially and is zoomed in at a selected edge in Figure 3(d). The GGVF vectors are projected onto the slice. The GGVF force points to the edges and therefore drives the nuclear boundaries toward the PEV edges. 


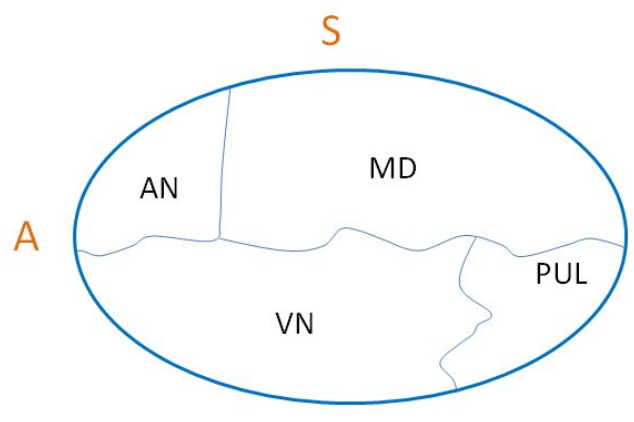

(a)

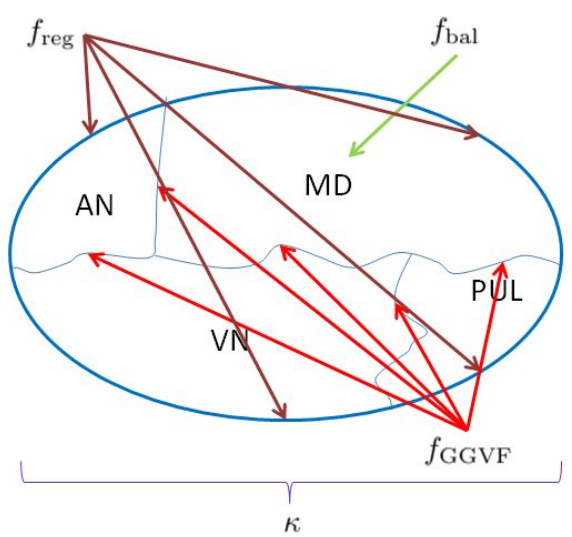

(b)

Figure 2. A diagram of (a) the relative relationship of each nuclei to segment; and (b) the forces on each boundary.

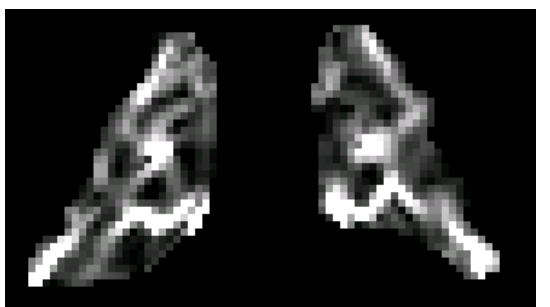

(a)

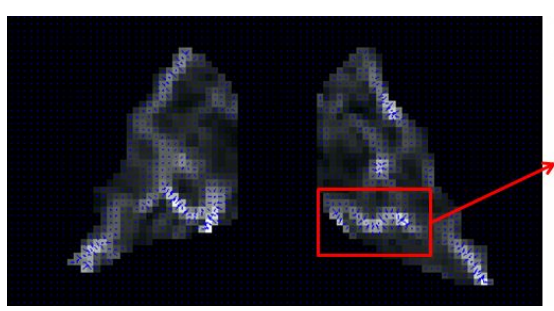

(d)

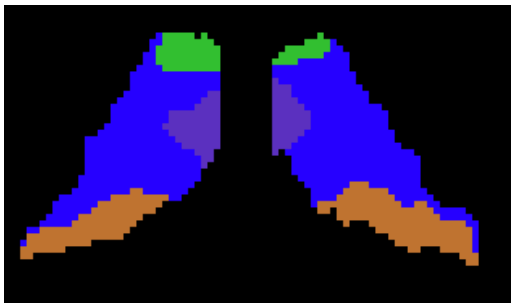

(b)

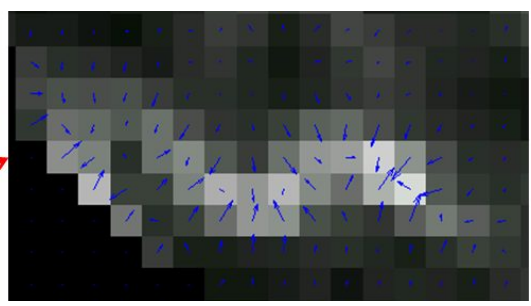

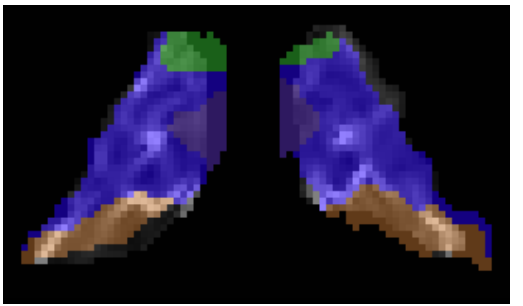

(c)

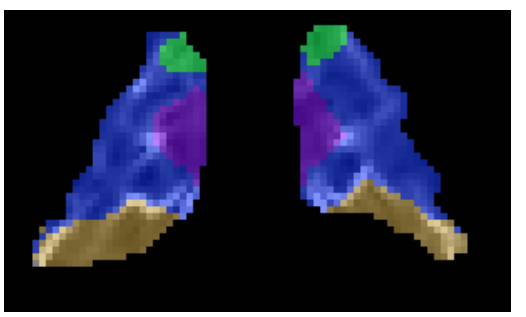

(e)

Figure 3. Examples of (a) the edge map; (b) the initialization; (c) the initialization overlaid on the edge map; (d) the GGVF overlaid on the edge map with a zoom-in view at a selected edge; and (e) the final segmentation overlaid on the edge map. Green: AN; purple: MD; blue: VN; yellow: PUL.

In addition to the evolution of the boundaries between different nuclei, we also need to move the nuclear boundaries so that the sum of all nuclei occupies the whole thalamus. A region based force is thus applied on the boundaries between each nucleus and the background using the thalamus mask. If a voxel $x$ on the boundary is inside the thalamus mask $I_{\text {thal }}$, the force expands the boundary; and if $x$ is outside the mask, the force makes the boundary shrink. Thus the region force at the voxel $x, f_{\text {reg:i,j }}(x)$, can be written as follows.

$$
f_{\text {reg: }: i, j}(x)=\left\{\begin{array}{l}
\alpha, \quad \text { if } i \text { or } j=\mathrm{BG} \text { and } x \in I_{\text {thal }} \\
-\alpha, \quad \text { if } i \text { or } j=\mathrm{BG} \text { and } x \notin I_{\text {thal }} \\
0, \quad \text { otherwise }
\end{array}\right.
$$

Apart from the forces above, we observe that for the $\mathrm{MD}$, it is necessary to apply a small balloon force on the nucleus to grow it close enough to the edges to be influenced by the GGVF force. Without such a force, the MD sometimes stays still or even shrinks due to the curvature force. Therefore, a balloon force is applied 
specifically on the MD as below.

$$
f_{\mathrm{bal}: i, j}(x)= \begin{cases}\beta, & \text { if } i=\mathrm{MD} \quad \text { or } \quad j=\mathrm{MD} \\ 0, & \text { otherwise }\end{cases}
$$

Finally, a curvature force $\kappa$ is used on every boundary as a smoothing force.

\section{EXPERIMENTS}

\subsection{Data Acquisition and Pre-processing}

The MP-RAGE and diffusion weighted images (DWI) were obtained on a 3T MR scanner (Intera, Philips Medical Systems, Netherlands). The DWI were acquired using a multi-slice, single shot EPI sequence. Each sequence had one b0 image and used 30 gradient directions. The DWI were then co-registered to corresponding MP-RAGE images. Both of them were resampled to the resolution of $0.828125 \mathrm{~mm}$ isotropic. The diffusion tensors were estimated using CATNAP. ${ }^{13}$

\subsection{MGDM Results}

Experiments were carried out on 5 subjects (3 patients and 2 controls). A 3D rendering of a result is shown in Figure 4. The cross section on a representative slice is overlaid on the edge map in Figure 3(e), where we can see that the boundaries between nuclei sit on the edges. Furthermore, the contours of each nucleus is overlaid on the MP-RAGE in Figure 5(a), where it can be confirmed that conventional anatomical MR images such as MP-RAGE do not provide sufficient contrast for the thalamic parcellation; the contours are then shown with the PEV's projected onto the plane in Figure 5(b) to show the homogeneity in the segmented nuclei. It should be noted that the VN is a large nuclear group that is composed of smaller nuclei such as the ventral anterior, the ventral lateral, and the ventral posterior nucleus. Thus the orientations of the PEV's in the VN are more heterogeneous than in the other three nuclei.
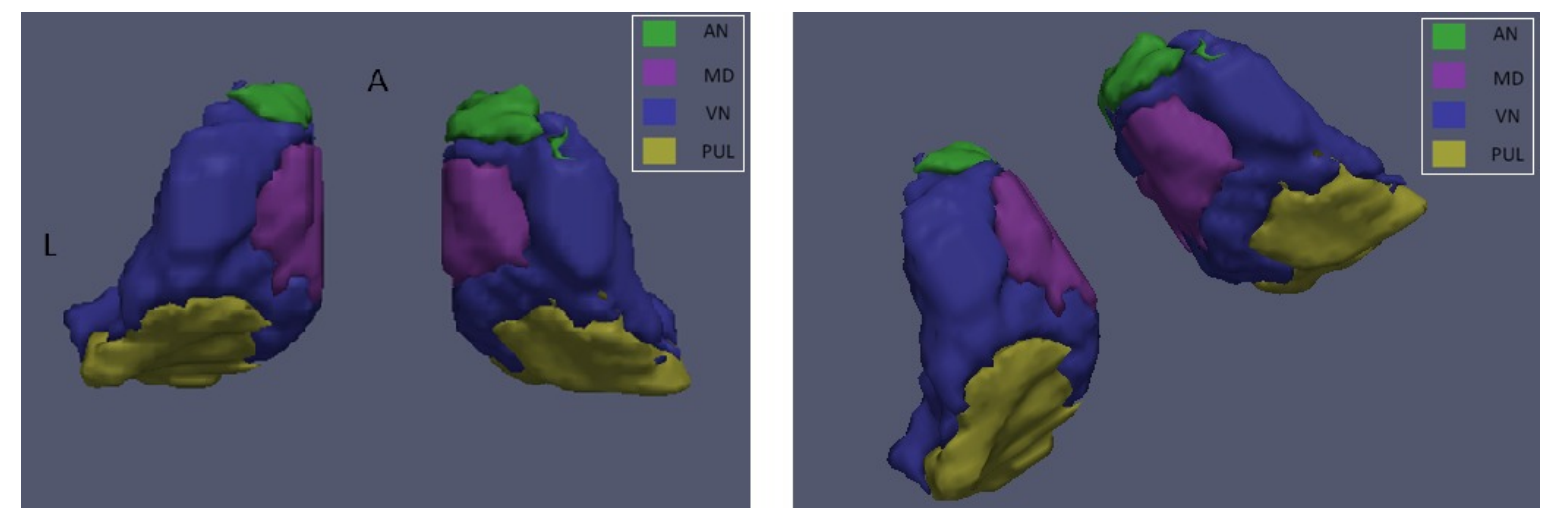

Figure 4. A 3D rendering of a parcellation result. Left: axial view; right: oblique view.

We have also made quantitative comparison with manual delineations generated according to the protocol mentioned previously. ${ }^{11}$ Note that although both patients and controls are included in the test, we do not discriminate between them in the analysis of our method. The Dice coefficient is computed using the following formula.

$$
D=\frac{2|X \cap Y|}{|X|+|Y|}
$$

where $X$ represents our results and $Y$ stands for the manual delineations, and $|\cdot|$ stands for the volume. The average surface distance (ASD) was also calculated, and is shown together with the Dice result in Table 1. The Dice coefficient indicates a good overlap with the manual segmentations; and the ASD shows that the boundaries of our results are close to those of the manual results. Moreover, in the work of Fan et al., ${ }^{11}$ it was reported that 


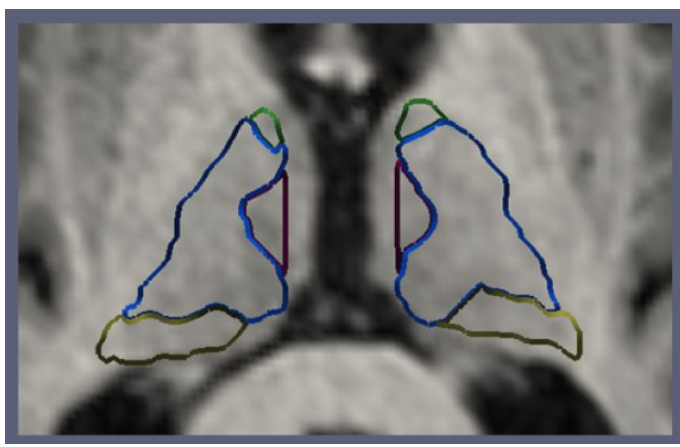

(a)

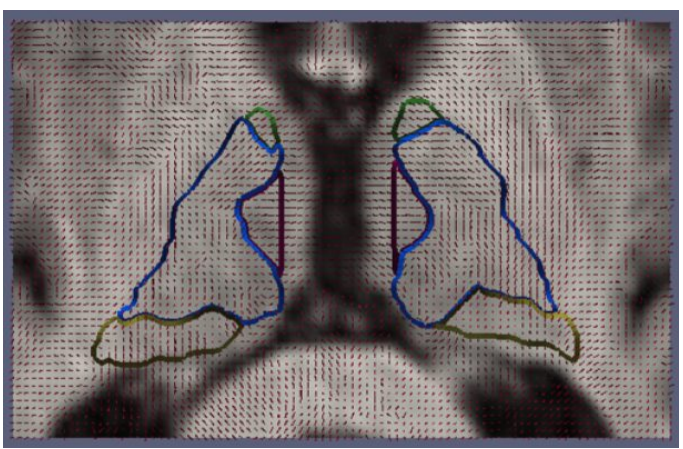

(b)

Figure 5. A cross section of the segmentation results: the contours of each nucleus (a) overlaid on the MP-RAGE; and (b) shown with the PEV's. Green: AN; purple: MD; blue: VN; yellow: PUL.

the average Dice coefficients between the four raters for the AN, MD, VN, and PUL were 0.323, 0.644, 0.763, and 0.556. Compared with these numbers, the average Dice coefficients in Table 1 shows that our segmentations are comparable with the manual work. Some of the numbers are much better partly because we use the same thalamus mask obtained from the manual delineation. In sum, the numbers demonstrate that the parcellation results agree with the manual delineations.

Table 1. The Dice coefficient (left) and ASD (mm) (right) between the parcellated nuclei and manual delineations

\begin{tabular}{c|cccc} 
(Dice) & AN & MD & VN & PUL \\
\hline Subject 1 & 0.664 & 0.707 & 0.869 & 0.822 \\
Subject 2 & 0.712 & 0.736 & 0.869 & 0.819 \\
Subject 3 & 0.782 & 0.703 & 0.896 & 0.870 \\
Subject 4 & 0.736 & 0.771 & 0.859 & 0.794 \\
Subject 5 & 0.737 & 0.662 & 0.878 & 0.765 \\
\hline Average Dice & 0.726 & 0.716 & 0.874 & 0.814
\end{tabular}

\begin{tabular}{c|cccc}
$($ ASD) & AN & MD & VN & PUL \\
\hline Subject 1 & 0.703 & 0.832 & 0.565 & 0.571 \\
Subject 2 & 0.718 & 0.676 & 0.590 & 0.495 \\
Subject 3 & 0.435 & 0.679 & 0.467 & 0.361 \\
Subject 4 & 0.537 & 0.777 & 0.576 & 0.652 \\
Subject 5 & 0.565 & 0.894 & 0.601 & 0.609 \\
\hline Average ASD & 0.592 & 0.771 & 0.560 & 0.537
\end{tabular}

\section{CONCLUSION}

In this work, we presented a method for the thalamic parcellation. An MGDM framework is used with the information of the fiber orientation, which is represented by the PEV. Since the PEV is an orientation, we map it into the 5D Knutsson space to eliminate the directional ambiguity and then calculate the edge map. Then the GGVF force is obtained from the edge map to drive the boundaries between the nuclei, together with other refining forces. Experiments on real subjects measuring the Dice coefficient and the average surface distances show that our results agree with the manual delineations of the nuclei. Future work will include the application of the method on a larger cohort and will include a finer parcellation involving more thalamic nuclei.

\section{Acknowledgments}

This work is partially supported by NIH/NINDS 2R01NS056307-06A1 and the China Scholarship Council.

\section{REFERENCES}

[1] Herrero, M.-T., Barcia, C., and Navarro, J., "Functional anatomy of thalamus and basal ganglia," Child's Nervous System 18(8), 386 - 404 (2002).

[2] Cifelli, A., Arridge, M., Jezzard, P., Esiri, M. M., Palace, J., and Matthews, P. M., "Thalamic neurodegeneration in multiple sclerosis," Annals of Neurology 52(5), 650 - 653 (2002). 
[3] Behrens, T. E. J., Johansen-Berg, H., Woolrich, M. W., Smith, S. M., Wheeler-Kingshott, C. A. M., Boulby, P. A., Barker, G. J., Sillery, E. L., Sheehan, K., Ciccarelli, O., Thompson, A. J., Brady, J. M., and Matthews, P. M., "Non-invasive mapping of connections between human thalamus and cortex using diffusion imaging," Nature Neuroscience 6(7), $750-757$ (2003).

[4] Wiegell, M. R., Tuch, D. S., Larsson, H. B., and Wedeen, V. J., "Automatic segmentation of thalamic nuclei from diffusion tensor magnetic resonance imaging," NeuroImage 19(2), 391 - 401 (2003).

[5] Duan, Y., Li, X., and Xi, Y., "Thalamus segmentation from diffusion tensor magnetic resonance imaging," Journal of Biomedical Imaging $\mathbf{2 0 0 7}(2), 1$ - 5 (2007).

[6] Jonasson, L., Hagmann, P., Pollo, C., Bresson, X., Wilson, C. R., Meuli, R., and Thiran, J.-P., "A level set method for segmentation of the thalamus and its nuclei in DT-MRI," Signal Processing 87(2), 309 - 321 (2007).

[7] Rittner, L., Lotufo, R., Campbell, J., and Pike, G., "Segmentation of thalamic nuclei based on tensorial morphological gradient of diffusion tensor fields," in [Biomedical Imaging: From Nano to Macro, 2010 IEEE International Symposium on], 1173 - 1176 (2010).

[8] Ziyan, U., Tuch, D., and Westin, C.-F., "Segmentation of thalamic nuclei from DTI using spectral clustering," in [Medical Image Computing and Computer-Assisted Intervention - MICCAI 2006], Lecture Notes in Computer Science 4191, 807 - 814 (2006).

[9] Fan, X., Bazin, P.-L., Bogovic, J., Bai, Y., and Prince, J., "A multiple geometric deformable model framework for homeomorphic 3D medical image segmentation," in [Computer Vision and Pattern Recognition Workshops, 2008. IEEE Computer Society Conference on], 1 - 7 (2008).

[10] Knutsson, H., "Producing a continuous and distance preserving 5-D vector representation of 3-D orientation," in [IEEE Computer Society Workshop on Computer Architecture for Pattern Analysis and Image Database Management (CAPAIDM)], 175 - 182 (1985).

[11] Fan, X., Thompson, M., Bogovic, J., Bazin, P.-L., and Prince, J., "A novel contrast for DTI visualization for thalamus delineation," in [Proceedings of SPIE Medical Imaging], 7625 (2010).

[12] Xu, C. and Prince, J., "Generalized gradient vector flow external forces for active contours," Signal Processing 71(2), 131 - 139 (1998).

[13] Landman, B., Farrell, J., Patel, N.-L., Mori, S., and Prince, J., "DTI fiber tracking: the importance of adjusting DTI gradient tables for motion correction. CATNAP - a tool to simplify and accelerate DTI analysis," in [Proc. Org Human Brain Mapping 13th Annual Meeting], (2007). 